\title{
Chronic Ovarian Pain as Illustrated in the Record of a Case finally Treated by Double Oophorectomy.*
}

\author{
Bу A. W. Russell, M.A., M.B.
}

WHEN this communication was postponed from the last meeting of the Society it was suggested that it might be made the basis of a short discussion on the operation of oopphorectomy for the relief of pain and its after-results.

Abdomino-pelvic pain in women provides both the physician and the surgeon with some of the most complicated and puzzling problems in medicine, and its various aspects and relations might profitably monopolize our attention for the whole evening. In this short paper I am limited to the class of cases that are perhaps the most difficult to understand, while they are usually the most unsatisfactory to treat - the cases of chronic so-called ovarian pain without obvious lesion discoverable by ordinary methods of examination.

Chronic ovarian pain may be defined as the persistent pain felt in its greatest intensity at or near a point on the abdominal wall about two inches or three fingerbreadths horizontally inwards from the anterior-superior spine, limited to an area corresponding to the tenth dorsal segment, and not associated with any recognizable lesion or abnormality of any of the abdominal organs. This pain must be accepted clinically as a distinct entity in spite of the criticisms of the gynæcologist who drove long needles through that part in the cadaver and frequently missed the ovary, or of the neurologist who said that he found the same localized tenderness in a hysterical male.

Pain limited to this ovarian region may be associated with disease of any of the other abdominal organs-bowel, liver and gall-bladder, kidney and ureter, or another part of the generative tract itself, and operations have sometimes been begun for its relief only to discover this fact, but such a mistake becomes less and less likely as knowledge advances and experience increases. Our real difficulties begin when we have to discriminate between simple chronic ovarian pain and pain felt in neurasthenia or in hysteria. With careful and repeated observation and examination, helped if necessary by an anæsthetic, the diagnosis will usually be possible. Gross lesions in the pelvis, or in other abdominal organs, will be ascertained by pelvic examination and by other clinical methods; the presence of peritonitis will be marked by its wider and more

* Read at a meeting of the Glasgow Obstetrical and Gynocological Society held on 22nd May, 1912. 


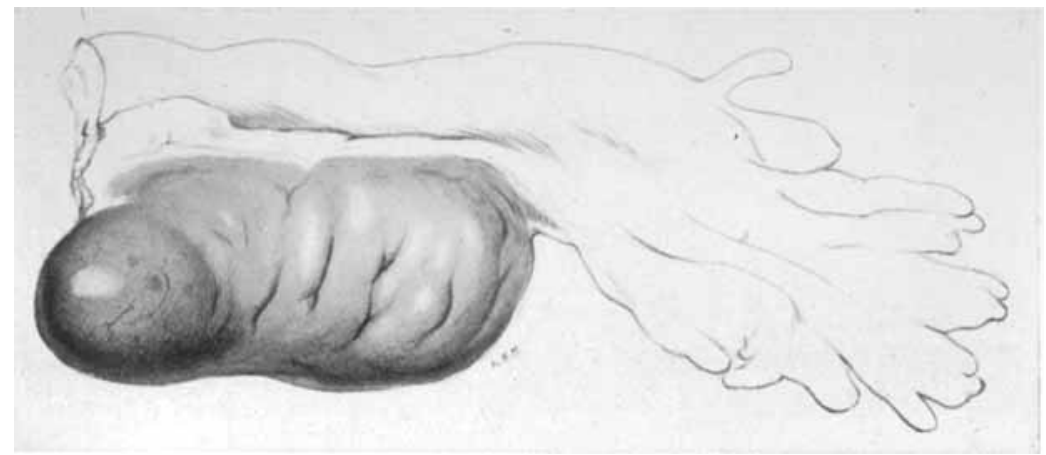

Right Ovary from Behind (Natural Size).

Showing distended follicle and corrugation.

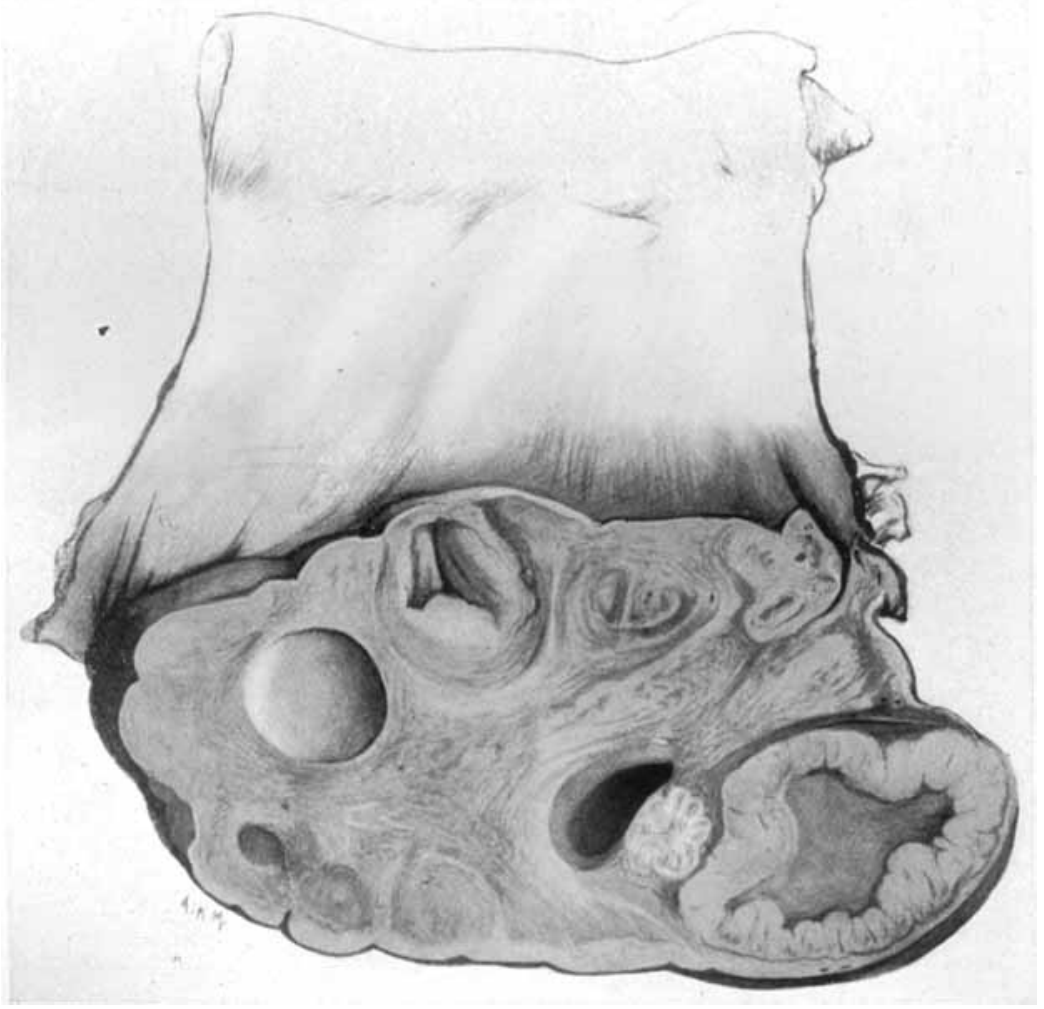

Right Ovary in Section ( $\times 2)$.

Showing sclerosis, unruptured cysts, and thickened cortex and cyst wall. 
irregular distribution, and will not be limited to the tenth dorsal segment; neurasthenia, a frequent sequel to chronic ovarian pain, has its own characteristic symptoms, and may need treatment after removal of the source of the pain; hysteria, as known to the modern physician, has its own definite physical signs upon which the diagnosis rests-its paræsthesia and hyperæsthesia and other nerve disturbances, and the absence of limit of the pain to a definite segment of the nervous system, though it adds its own difficulty to the treatment by the concentration of the mind on the local ovarian pain when present as a complication.

When we have excluded all these elements we have still a proportion of cases, and these often the most persistent and aggravated in their character, which are associated with nothing else but a condition of the ovaries not readily recognizable by the ordinary methods of examination, and seldom relieved by anything short of operative treatment. To this class belongs the case that is the reason for my present communication.

Mrs. N. consulted me about the end of 1908 , nearly three and a half years ago. She was then 37 years of age, her husband was living, and she had a healthy boy 10 years old. Menstruation began early, and before her marriage had always been painful; but she had relief in this respect after marriage, and after the birth of her child she was in good health. Subsequently she had two miscarriages about the third month, and was curetted some time after the first but not at the time of the second nor after it. Her abdominal pain and deterioration of health are assigned to these incidents. Tamponade, frequent douching, pessaries, applications of iodine, restful holidays in the country, etc., were tried, with no apparent benefit, but rather with progressive increase of the pain. Her condition had become so serious that five months before I was asked to see her there was a consultation, and as a result she was put upon a strict regimen of treatment, including four daily douches, each of four pints of hot water, special stress being laid upon their regularity and the quantity of fluid used. I mention these details to show that if ever such a case could be cured by medical means most carefully prescribed and as faithfully carried out, this ought to have been.

At the time that I saw her first she was in a miserable state of physical and mental health, and would not admit the slightest benefit from the elaborate and prolonged ritual of treatment which she had religiously followed for the previous five or six months. She complained of constant wearing pain, specially marked on the right side, unfitting her for her domestic duties, and making life itself a burden. The uterus was found to be slightly enlarged, and there was acute tenderness over the ovarian regions, this being specially marked at a rounded projection on the right ovary corresponding to a 
distended follicle with thickened wall seen in the specimen. The persistence, and even the gradual aggravation, of her symptoms, left me little hope that I could help her by any variation of the means of medical treatment. An examination under an anæsthetic gave no fresh light on the case, so I obtained permission for a radical operation if this was found to be necessary.

The operation needs no description. Both ovaries and tubes were removed, primary convalescence from the operation was uncomplicated, and the patient was back in her own home within four weeks. But this is not the whole story. Complete recovery of health is naturally a slow process in such cases, and this patient, by one or two sharp experiences after over-exertion or prolonged fatigue in domestic work, discovered that a modern operation was not the exact equivalent of an ancient miracle. Though $I$ had given a guarded prognosis, I was myself for a time anxious as to the final result. Three months after the operation she was still easily tired, and there was occasional pain in the left side. Eight months after the operation, however, nutrition had markedly improved. Her face had a younger look, and no pain could be elicited either on palpation over the ovarian regions, previously so tender as to hardly bear touching, or by vaginal examination.

Some one has said that "no conclusions as to the value of operative treatment in curing the so-called ovarian pain can safely be drawn except from cases watched for a long time subsequent to operation." I have waited three years to report this case, and have in the interval had the satisfaction of seeing my patient (who seemed doomed to invalidity, if not something worse) regain her health and resume her normal habits or life, to her own intense gratification and the delight of her friends.

I may appear to some to be making too much of a single case, but it is not the only one in my experience, and sometimes the exposition of one case in all its details may be quite an educative and as effective an argument as a big series of cases not entirely alike in their characteristies. I am reminded of two other cases, one of them, I think, the first double oöphorectomy that I did for chronic ovarian pain, the other my most recent ease of this, in whom, however, I left a very small part of one ovary.

The first, a single woman of 30-35 years, made a good recovery, but owing to an accident at the operation developed about three weeks later a double phlegmasia, and for months after had persistent distressing pain high in the abdomen, necessitating a second abdominal section. In spite of all her subsequent suffering she gave the repeated assurance that she had entirely lost the original ovarian pain.

The last was a young married woman of 28 years, who had had one child five years ago. Her ovaries show the same characters as 
the other specimens. Her pain dates back to her confinement, and has latterly been constant, with acute prostrating exacerbations. From the day of the operation, five weeks ago, this patient has declared that she has becn completely relieved of her pain. And this relief is explained by some to be due to "suggestion !"

The pathological bearings of the case have their own interest, and form part of the reason for my report. Ovaries at different ages, and in different persons at the same age, vary in appearance and structure so greatly that it is difficult to establish either a healthy or a pathological standard for comparison of specimens. The ovaries of my patient and the drawings of Mr. Maxwell, as well as the microscopic sections, seem to me to show undue corrugation of their surfaces, abnormal thickening both of the cortex and of the walls of unruptured follicles - a condition of cystic solerosis. I am convinced that for so advanced a pathological change the only likely operation is oöphorectomy, and I could not hesitate to operate on both sides if the lesion is bilateral and advanced.

It is difficult to understand how such a diseased process can be delayed or arrested in its progress, the original cause being as yet undiscovered. Just as the condition of fibrosis of the uterus is attributed to a toxic influence, we may have a similar agency at work here. In the earlier stages much may be done by improving and maintaining the general health, and so avoiding or delaying the distressing complication of hysteria or neurasthenia. When intelligent palliative treatment has been well tried, and has failed, there should then be no delay in operating.

A small abdominal incision allows the operator to inspect the pelvis, and he has then the choice of several operations. He may excise the more discased portion or portions, leaving room for the development of other follicles in the healthy parts; or he may adopt the alternative of decapsulation, or, more corrcetly speaking, decortication, of the ovary, which has been recommended and tried. Such operations are spocially desirable in the first resort in the younger patients. In advanced degeneration of the structures nothing short of complete oophorectomy is likely to relieve the sufferer. 